\title{
Using Wireless Sensor Networks to Achieve Intelligent Monitoring for High-Temperature Gas-Cooled Reactor
}

\author{
Jianghai Li, ${ }^{1}$ Jia Meng, ${ }^{2}$ Xiaojing Kang, ${ }^{2}$ Zhenhai Long, ${ }^{2}$ and Xiaojin Huang ${ }^{1}$ \\ ${ }^{1}$ Institute of Nuclear and New Energy Technology, Collaborative Innovation Centre of Advanced Nuclear Energy Technology, \\ Key Laboratory of Advanced Reactor Engineering and Safety of Ministry of Education, Tsinghua University, Beijing, China \\ ${ }^{2}$ Chinergy Co., Ltd., Beijing, China \\ Correspondence should be addressed to Xiaojin Huang; huangxj@tsinghua.edu.cn
}

Received 31 December 2016; Revised 12 April 2017; Accepted 26 April 2017; Published 30 May 2017

Academic Editor: Alejandro Clausse

Copyright (C) 2017 Jianghai Li et al. This is an open access article distributed under the Creative Commons Attribution License, which permits unrestricted use, distribution, and reproduction in any medium, provided the original work is properly cited.

\begin{abstract}
High-temperature gas-cooled reactors (HTGR) can incorporate wireless sensor network (WSN) technology to improve safety and economic competitiveness. WSN has great potential in monitoring the equipment and processes within nuclear power plants (NPPs). This technology not only reduces the cost of regular monitoring but also enables intelligent monitoring. In intelligent monitoring, large sets of heterogeneous data collected by the WSN can be used to optimize the operation and maintenance of the HTGR. In this paper, WSN-based intelligent monitoring schemes that are specific for applications of HTGR are proposed. Three major concerns regarding wireless technology in HTGR are addressed: wireless devices interference, cybersecurity of wireless networks, and wireless standards selected for wireless platform. To process nonlinear and non-Gaussian data obtained by WSN for fault diagnosis, novel algorithms combining Kernel Entropy Component Analysis (KECA) and support vector machine (SVM) are developed.
\end{abstract}

\section{Introduction}

Wireless sensor network technology (WSN) can be employed in high-temperature gas-cooled reactors (HTGR) to achieve intelligent monitoring. Intelligent monitoring with WSN will not only reduce the direct cost of regular monitoring but also assist with various tasks, such as fault diagnosis and recovery, condition-based maintenance, and control of nuclear materials, which would optimize the operation and maintenance of the HTGR nuclear power plant (NPP).

WSN is a new technology in which interest is growing. Sensors with the wireless communication capability are able to cooperatively monitor physical or environmental conditions, such as temperature, pressure, sound, and vibration. A sensor can be a fully contained device with a sensing component, a radio transceiver, a microcontroller, and an energy source all in the same module. It can also be a conventional instrument attached to a wireless data transmitter, where sensed data are transmitted to a data processing center wirelessly. The WSN has been widely used in industry, agriculture, military, transportation, and other fields.
At present, regular monitoring of NPPs mainly employs wired connections between sensors and data processing centers. For example, in Instrumentation and Control (I\&C) systems, instrument signals are transmitted by hard wires. Although the wired connection is assumed to be more reliable, it is costlier and increases the complexity of plant layout. A rough estimate of the cost of normal wiring in an NPP is around US $\$ 2,000$ per $0.3 \mathrm{~m}$, whereas the cost of using wireless technology for the same application is less than US $\$ 20$ per $0.3 \mathrm{~m}$ [1]. Moreover, increasing the number of cables also increases the number of cable trays required, which occupy a lot of space and complicate design and construction.

Applying WSN in the monitoring part of equipment and processes in HTGR NPP can effectively alleviate the above problems. The replacement of some wired cables with wireless connections will create economic benefits for HTGR by cutting the cost of wiring, reducing the space required for cable trays, and shortening the period of design and construction for the cable tray layout.

A WSN can also enable higher levels of monitoring. With WSNs, massive and heterogeneous data about equipment 
and processes can be gathered. These rich monitoring data offer brand new possibilities for better fault detection and diagnosis, more advanced maintenance, and more efficient management of nuclear materials.

To achieve such high levels of intelligent monitoring, three questions must be answered.

Q1. What are intelligent monitoring schemes for various applications? The monitoring scheme includes the selection of process variables and equipment parameters to be monitored, the placement of wireless sensors, and the power supply for sensors and transmitters.

Q2. How can large amounts of heterogeneous data be transmitted via the WSN? The wireless communication problems for intelligent monitoring in HTGR include the selection of appropriate communication protocols, as well as the electromagnetic compatibility concern and the security issue.

Q3. How should we deal with the large scale of data to realize intelligent monitoring?

This paper is organized as follows. The kind of data that should be monitored (see Q1) depends on different intelligent monitoring schemes for various applications. Three monitoring schemes in three scenarios are proposed in Section 2. The methods of transmitting data in Q2 are specified in wireless communication protocols. In Section 3, wireless protocols of WSNs are compared and some important issues are discussed. We also demonstrate specific data processing algorithms related with Q3 in Section 4. Conclusions are drawn in Section 5.

A preliminary version of this work has been published in [2] to deal with the intelligent monitoring for general NPPs. Two data processing algorithms of KPCA and SVM were proposed. In this paper, we focus on the intelligent monitoring for HTGR. We make more detailed monitoring schemes that are specific for HTGR. Additional material is supplemented to adequately address three major concerns of the wireless communication. We further develop the KECASVM algorithms for slow process fault diagnosis of HTGR.

\section{Intelligent Monitoring Schemes for HTGR}

Intelligent monitoring with rich information about processes and equipment will greatly benefit the safety and cost efficiency of HTGR. Currently, the number and types of monitoring variables are determined based on the aim of controlling performance during normal operation. Insufficient consideration is given to other aspects of the full life cycle of NPPs, such as off-normal operation, maintenance, and retirement. To realize the type of intelligent monitoring that can optimize the full life cycle of a NPP, massive and heterogeneous data are required [3]. Multiple types of data out of the scope of conventional monitoring can be incorporated into intelligent monitoring with a WSN, such as radiofrequency identification (RFID) data, video camera data, and microphone sensor data. Some examples will be discussed below to show how rich information could contribute to advanced maintenance, fault diagnosis and recovery, and management of nuclear materials.

Condition-based maintenance requires comprehensive monitoring of equipment. At present, only a few critical parameters of equipment are monitored by I\&C systems due to the limited number of wired input channels. The number of wired input channels is constrained not only by the cost of monitoring modules in the data center but also by the confined space occupied by sensor wiring [4]. The WSN can break the limitation of the cost and the space. The wireless sensors are able to collect abundant sensing data and send them to the data center via wireless channels. This can be achieved without significantly increasing the number of monitoring modules or expanding wiring space, since one access point can gather data from multiple sensors via wireless technology. For example, we can consider the task of monitoring the main helium blower of HTGR. In addition to critical parameters like rotational speed and the pressure rise, diverse information about the equipment, such as the vibration of the base, the surface temperature distribution, and even the operational noise, could help in assessing the need for maintenance. To gather this diverse information, a variety of wireless sensors could be positioned around the main helium blower motor. Several infrared thermometers placed above the blower can observe the temperature distribution of its surface. A number of vibration sensors laid on the base of the blower can measure its vibration in multiple dimensions. A microphone array deployed beside the blower can collect data on operational noise in an uninterrupted manner. All these heterogeneous observations would be sent to the data center to enable the real-time health monitoring of the main blower.

Another example shows fault diagnosis and recovery with wireless monitoring data. The feed water flow in a HTGR measured by a conventional flowmeter can be sent back to the process control system via wired cables or via a wireless transmitter. The wireless data serves as a diverse backup for the wired data. The backup data can verify the wired data to detect malfunctions of cables or monitoring modules. If the failure is caused by communication faults, regardless of whether this occurs in the wired network or in the wireless channel, the checksum transmitted along with the data will indicate the error. If the fault is identified in the wired network, the valid wireless data can be used in place of the faulty wired data to maintain continuous monitoring.

The third example demonstrates how the RFID technology and wireless networks can contribute to efficient management of spent fuel from HTGR. The canister-based dry spent fuel storage system is adopted in HTGR [5]. Each canister has a capacity of 40000 spent fuel spheres and can be placed in the spent fuel storage building with concrete shields. In current design, the ID is carved on the cap of the canister and is read by the operator through cameras. However, cameras at fixed positions have difficulty reading the ID at the first stage of the canister transport, which reduces the efficiency of spent fuel management. RFID based on wireless networks provides an alternative for the conventional optical ID. An RFID tag is composed of memory, modulator, and wireless antenna. They can be attached on any nuclear material containers 
or packages that require rigorous monitoring. Information about nuclear material can be recorded and updated on the RFID tags. Using an RFID reader, the information on tags can be read in a contactless way through the whole transport process of spent fuel canisters. Meanwhile, the positions of nuclear material can be localized and tracked by RFID tagreader pairs. The up-to-date information retrieval and the localization by RFID can not only enhance the efficiency of nuclear material management but also reduce risk of nuclear material loss.

\section{Wireless Transmission for the WSN of HTGR}

To employ wireless technologies to transmit monitoring data in HTGR, three major concerns need to be considered comprehensively. They are wireless devices interference, cybersecurity of wireless networks, and wireless standards selected for wireless platform of HTGR.

For the first concern, wireless devices must be electromagnetically compatible (EMC) with the existing I\&C systems. The power level of wireless sensors is usually below $20 \mathrm{~mW}$. In the EMC standard of IEC 61000-4-3 [6] (GB/T 17626.3), note 4 in Section 5.2 states that "other systems operating in this frequency range, e.g. radio LANs operating at $2.4 \mathrm{GHz}$ or higher frequencies, are generally very low power (typically lower than $100 \mathrm{~mW}$ ), so they are much less likely to present significant problems." Even so, in order to ensure that there is no interference by wireless devices, all the I\&C systems, in particular the safety-related systems, should pass the susceptibility testing in the frequency range covering the wireless frequency band. The Regulatory Guide 1.180 [7] suggests that this susceptibility test operating envelope will remain the same as at lower frequencies than $2.4 \mathrm{GHz}$.

For the second point, cybersecurity is another major concern with using wireless sensors in NPPs. It is believed that unprotected wireless transmission in opening space makes the network platform vulnerable to external malicious cyber-attacks, such as spoofing and jamming. With the advanced technologies of encryption, authentication process, channel fault-tolerance, and security certification, the cybersecurity concern of wireless network can be significantly allayed [8]. Moreover, for the intelligent monitoring applications of the HTGR with inherent safety feature, the cyber-attacks pose little potential risk to the plant. In addition, it should be mentioned that the cybersecurity features cannot protect against attacks at the physical layer. The physical security requirement of the wireless platform should be the same as that of the wired I\&C system.

For the third concern, the selection of wireless standards is the main task of wireless platform design. More and more applications have been or are going to be built on the wireless platform of NPPs, such as voice communication, personnel positioning, and radiation monitoring. The requirements of future wireless applications need to be taken into account in advance. Thus, the major criteria for the wireless platform of HTGR are listed as follows: (i) High bandwidth

(ii) Lower RF power while covering a wide range

(iii) Being scalable and extensible

(iv) Widespread support by industry

The first criterion, high bandwidth, provides the possibility to accommodate high traffic applications such as voice and video. The second criterion intends to balance the emission restriction with the deployment cost. The third one and the fourth one consider the long-term utility of the wireless platform.

According to the result of our research, wireless standards which have been employed in NPPs in China include WiFi, McWill, PHS, and TDD (Table 1). Some other potential wireless standards using IEEE 802.15.4 [1], such as ZigBee, WirelessHART, and ISA100, which have low data transmission rate, are not taken into consideration for the wireless platform of HTGR. Currently, the wireless applications are limited to voice communications. The great potential of wireless data communication for the intelligent monitoring has not been fulfilled yet. Only McWill and Wi-Fi technologies have the potential for the data communication. They have the capabilities of high bandwidth, high security, and terminal roaming handoff. However, McWill causes higher RF power and thus only allows one-way communication for mobile receivers in the nuclear island [9]. Wi-Fi technology with $2.4 \mathrm{GHz}$ band meets all the above requirements of wireless applications in NPPs. The high bandwidth of communication, the openness of protocols, and the scalability of the systems make the Wi-Fi suitable for a variety of applications, such as wireless monitoring, emergency communications, and mobile operation.

\section{Data Processing Algorithms for Intelligent Monitoring}

In this section, we aim to provide solution based on WSN data for a particular fault diagnosis problem of HTGR, that is, slow process fault diagnosis. The slow process faults mainly refer to the faults that occur slowly over time, such as leakage and small broken pipe of helium gas. Unlike the failures of main components within the system, such as the main helium blower and turbine, these failures are not easily noticed due to the compensation control and the simple limit alarming. However, slow process faults can also lead to the degradation of reactor performance or even abnormal operational occurrence. The difficulties in slow process fault diagnosis, including fault detection and fault classification, are the nonlinear and non-Gaussian nature of the monitoring data obtained by WSN.

There have been many fault detection methods, such as PCA and its variants. PCA performs poorly for the nonlinear problem, as it assumes that the process data are linear. To overcome the shortcoming of the PCA, several nonlinear extensions of PCA were reported, like Kernel PCA (KPCA). KPCA was used to detect faults in two example systems in $[10,11]$. The problem of fault detection was addressed in mechanical systems using a KPCA-based 
TABLE 1: Comparison of wireless standards in NPPs.

\begin{tabular}{|c|c|c|c|c|}
\hline \multirow{2}{*}{ Spec. } & \multicolumn{3}{|c|}{ Tech. } & \multirow[b]{2}{*}{ TDD } \\
\hline & McWill & $\mathrm{Wi}-\mathrm{Fi}$ & PHS & \\
\hline Frequency band & $\begin{array}{c}1875-1805 \mathrm{MHz} \\
\text { Need licenses }\end{array}$ & $\begin{array}{c}2.4 \mathrm{GHz} \\
\text { Free band }\end{array}$ & $\begin{array}{c}1900-1920 \mathrm{MHz} \\
\text { Need licenses }\end{array}$ & $\begin{array}{c}800 \mathrm{MHz} \\
\text { Need licenses }\end{array}$ \\
\hline Bandwidth & $15.36 \mathrm{Mbps}$ & $300 \mathrm{Mbps}$ & 64/128 Kbps & $64 \mathrm{Kbps}$ \\
\hline $\begin{array}{l}\text { Transmitting } \\
\text { power }\end{array}$ & $\begin{array}{c}\text { Base station } 30 \mathrm{~W} \\
\text { (outdoor) } \\
\text { Base station } 10 \mathrm{~mW} \\
\text { (indoor) } \\
\text { Cellphone } 2 \mathrm{~W} \\
\text { (outdoor) } \\
\text { Cellphone } 10 \mathrm{~mW} \\
\text { (indoor) } \\
\end{array}$ & $\begin{array}{l}\text { Base station } 0.1 \mathrm{~W} \\
\text { (indoor) } \\
\text { Base station } 0.5 \mathrm{~W} \\
\text { (outdoor) } \\
\text { Cellphone } 0.1 \mathrm{~W}\end{array}$ & $\begin{array}{l}\text { Base station } \\
10 \mathrm{~mW} \\
\text { Cellphone } \\
10 \mathrm{~mW}\end{array}$ & $\begin{array}{c}\text { Base station } 0.6-40 \mathrm{~W} \\
\text { Cellphone } 1.8 \mathrm{~W}\end{array}$ \\
\hline $\begin{array}{l}\begin{array}{l}\text { Coverage } \\
\text { (outdoor) }\end{array} \\
\end{array}$ & $4 \mathrm{Km}$ & $0.5 \mathrm{Km}$ & $0.5 \mathrm{Km}$ & $5 \mathrm{Km}$ \\
\hline Mobility & $120 \mathrm{Km} / \mathrm{h}$ & $50 \mathrm{Km} / \mathrm{h}$ & $40 \mathrm{Km} / \mathrm{h}$ & $100 \mathrm{Km} / \mathrm{h}$ \\
\hline Advancement & Better & Great & Normal & Good \\
\hline Security & $\begin{array}{l}\text { Encryption, group } \\
\text { function, authorization, } \\
\text { channel fault-tolerance, } \\
\text { and security certification }\end{array}$ & $\begin{array}{l}\text { Encryption, group } \\
\text { function, } \\
\text { authorization, } \\
\text { channel } \\
\text { fault-tolerance, and } \\
\text { security certification }\end{array}$ & AI encryption & Authorization, $\mathrm{P} 2 \mathrm{P}$ encryption \\
\hline $\begin{array}{l}\text { Industrial } \\
\text { cluster }\end{array}$ & Scarcely & $\begin{array}{c}\text { More than } 300 \\
\text { alliance members }\end{array}$ & Weed out & TETRA/PDT/GT800/GoTa/iDEN \\
\hline Functionality & $\begin{array}{c}\text { Dispatcher } \\
\text { voice/note/data/real- } \\
\text { time } \\
\text { video }\end{array}$ & $\begin{array}{c}\text { Dispatcher } \\
\text { voice/note/data/HD } \\
\text { real-time } \\
\text { video/location }\end{array}$ & Voice/note & Voice/note \\
\hline
\end{tabular}

method [12]. In [13], the methods of KPCA and kernel partial least analysis were used for fault diagnosis and process monitoring of a continuous annealing process and fused magnesium furnace. However, non-Gaussian information is usually included in the high-dimensional data of the industry process; these fault detection methods no longer fit well. In view of the nonlinear characteristics and non-Gaussian distributed data in HTGR, Kernel Entropy Component Analysis (KECA) algorithm for fault detection is demonstrated in Section 4.1.

For the fault classification problem, support vector machine (SVM), as a novel machine learning method based on statistical learning theory, has been widely employed [1417], as it is very suitable for the nonlinear data with small samples and high dimension. It gradually takes the place of neural network by using structural risk minimization principle instead of empirical risk minimization (ERM) principle. There are previous works on applying SVM to fault classification in many industrial areas. Four fault types of power transformer were identified by the trained multilayer SVM classifier [14]. A hybrid two-stage one-against-all SVM approach was proposed for fault diagnosis of defective rolling element bearings [15]. An improved SVM classifier was developed for fault diagnosis of actual analog circuits [16]. Combining chaos particle swarm algorithm with SVM, a novel fault diagnosis method was applied to isolate wireless sensor faults [17]. The KECA-SVM combining algorithm for the WSN data of HTGR is proposed in Section 4.2, which can overcome the nonlinear and non-Gaussian difficulties in slow process fault diagnosis.

4.1. KECA Algorithms for Fault Detection. As a new approach in reducing data dimension and detecting faults of HTGR, the Rényi quadratic entropy is first introduced by

$$
H(p)=-\log \int p^{2}(\mathbf{x}) d \mathbf{x},
$$

where $p(\mathbf{x})$ is the probability density function (PDF) of the data set $D=\mathbf{x}_{1}, \ldots, \mathbf{x}_{N}$. Since the logarithm is a monotonic function, what we only need to consider is the quantity $V(p)=\int p^{2}(\mathbf{x}) d \mathbf{x}$. To estimate $V(p)$ and then estimate $H(p)$, Parzen-window density estimation is given by

$$
\widehat{p}(\mathbf{x})=N^{-1} \sum_{\mathbf{x}_{t} \in D} k_{\sigma}\left(\mathbf{x}, \mathbf{x}_{t}\right),
$$

where $k_{\sigma}\left(\mathbf{x}, \mathbf{x}_{t}\right)$ is the Parzen window, also known as the kernel function, which centers on $\mathbf{x}_{t}$ with a width parameter $\sigma$. Here the most commonly used radial basis function is selected as the kernel function:

$$
k\left(\mathbf{x}, \mathbf{x}^{\prime}\right)=\exp \left(\frac{-\left\|\mathbf{x}-\mathbf{x}^{\prime}\right\|^{2}}{\left(2 \sigma^{2}\right)}\right) .
$$


Using (2) and the convolution theorem for Gaussian kernel function, we have

$$
\begin{aligned}
\widehat{V}(p) & =\int \widehat{p}^{2}(\mathbf{x}) d \mathbf{x} \\
& =N^{-2} \sum_{\mathbf{x}_{t} \in D} \sum_{\mathbf{x}_{t^{\prime}} \in D} \int k\left(\mathbf{x}, \mathbf{x}_{t}\right) k\left(\mathbf{x}, \mathbf{x}_{t^{\prime}}\right) d \mathbf{x} \\
& =N^{-2} \sum_{\mathbf{x}_{t} \in D} \sum_{\mathbf{x}_{t^{\prime}} \in D} k\left(\mathbf{x}_{t}, \mathbf{x}_{t^{\prime}}\right)=N^{-2} \mathbf{1}^{T} \mathbf{K} \mathbf{1},
\end{aligned}
$$

where $\left(t, t^{\prime}\right)$ th element of the $N \times N$ kernel matrix $\mathbf{K}$ is $k\left(\mathbf{x}_{t}, \mathbf{x}_{t^{\prime}}\right)$ and $\mathbf{1}$ is the $N \times 1$ column vector whose element is 1 . Thus, the Rényi quadratic entropy estimate can be compactly expressed as the form of kernel matrix. $\mathbf{K}$ can be eigendecomposed in the following form:

$$
\mathbf{K}=\mathbf{E D E}^{T} \text {, }
$$

where $\mathbf{D}$ is the diagonal matrix consisting of eigenvalues $\lambda_{1}, \ldots, \lambda_{N}$ and $\mathbf{E}$ is the matrix $\mathbf{e}_{1}, \ldots, \mathbf{e}_{N}$ whose column vectors are the corresponding eigenvectors. In the meantime, kernel matrix can be also written as the form of inner product matrix in kernel feature space:

$$
\mathbf{K}=\boldsymbol{\Phi}^{T} \boldsymbol{\Phi}=\mathbf{E D E}^{T},
$$

where $\boldsymbol{\Phi}=\mathbf{D}^{1 / 2} \mathbf{E}^{T}$. So $\widehat{V}(p)$ can be rewritten as follows:

$$
\widehat{V}(p)=N^{-2} \sum_{i=1}^{N}\left(\sqrt{\lambda_{i}} \mathbf{e}_{i}^{T} \mathbf{1}\right)^{2} .
$$

Each item in the above equation contributes to the entropy estimate. That is to say, certain eigenvalues and corresponding eigenvectors will contribute more to the entropy estimate than other eigenvalues and eigenvectors. Thus, in KECA algorithm, the eigenvalues and corresponding eigenvectors which are the first $l$ largest contribution to the entropy estimate are chosen. KECA can be interpreted as a data dimension reduction technique which projects $\Phi$ onto a subspace $U_{l}$ composed of those $l$ principal axes contributing more to the Rényi entropy estimate. The data transformation is formulated as follows:

$$
\Phi_{\mathrm{eca}}=P_{U_{l}} \Phi
$$

where $P_{U_{l}}$ is the loading matrix.

The $l$-dimensional matrix $\Phi_{\text {eca }}$ in kernel feature space can be also expressed as follows:

$$
\Phi_{\mathrm{eca}}=\mathbf{D}_{l}^{1 / 2} \mathbf{E}_{l}^{T}
$$

where $\mathbf{D}_{l}, \mathbf{E}_{l}$ are the matrices which consist of selected eigenvalues and corresponding eigenvectors, respectively.

Thereby, the new kernel matrix $\mathbf{K}_{\text {eca }}$ generated by KECA algorithm can be written as follows:

$$
\mathbf{K}_{\mathrm{eca}}=\boldsymbol{\Phi}_{\mathrm{eca}}^{T} \boldsymbol{\Phi}_{\mathrm{eca}}=\mathbf{E}_{l} \mathbf{D}_{l} \mathbf{E}_{l}^{T}
$$

Therefore, $-\log$ of the quantity $\widehat{V}_{l}(p)=\left(1 / N^{2}\right) \mathbf{1}^{T} \mathbf{K}_{\mathrm{eca}} \mathbf{1}$ can be considered as the Rényi entropy estimation of some data set which is denoted as $\mathbf{x}_{1}^{\prime}, \ldots, \mathbf{x}_{N}^{\prime} \cdot \widehat{V}_{l}(p)$ carries most of the information of the Rényi entropy estimation of the initial data set $\mathbf{x}_{1}, \ldots, \mathbf{x}_{N}$. Accordingly, data transformation $\mathbf{x} \rightarrow \mathbf{x}^{\prime}$ in input space is produced so that the entropy of $\mathbf{x}_{1}^{\prime}, \ldots, \mathbf{x}_{N}^{\prime}$ and the entropy of $\mathbf{x}_{1}, \ldots, \mathbf{x}_{N}$ are closely approximate. This also reflects data transformation of KECA in input space.

In contrast to KPCA which performs data transformation and dimension reduction by selecting eigenvalues and corresponding eigenvectors of the kernel matrix merely based on the size of the eigenvalues, KECA chooses the eigenvalues based on the contribution to entropy estimate.

To apply KECA algorithm to condition monitoring and fault diagnosis of HTGR, monitoring statistical control charts need to be constructed to reflect the operating condition. Here, Hotelling $T^{2}$ and SPE statistics are used to monitor the industrial process. Faults can be detected by judging whether $T^{2}$ and SPE statistics exceed the respective confidence limit or not. Faults occur as long as SPE statistic exceeds the confidence limit. If $T^{2}$ statistic exceeds the confidence limit and SPE statistic does not, working conditions may change.

The two measures are defined as follows. Hotelling $T^{2}$ statistic, which represents the major variation of the sample to the model, is the standard quadratic sum of principal component score vectors:

$$
T^{2}=\left[t_{1}, t_{2}, \ldots, t_{R}\right] \Lambda^{-1}\left[t_{1}, t_{2}, \ldots, t_{R}\right]^{T},
$$

where $R$ is the number of selected principal components.

SPE statistic, also known as the $Q$ statistic, which represents the goodness of fit of the sample to the built model, is the error between the actual measured variable and the KECA model:

$$
\mathrm{SPE}=\left\|\Phi(\mathbf{x})-\widehat{\Phi}_{R}(\mathbf{x})\right\|^{2}=\sum_{i}^{N} t_{i}{ }^{2}-\sum_{i}^{R} t_{i}{ }^{2},
$$

where $\widehat{\Phi}_{R}(\mathbf{x})$ is the reconstructed feature vector with principal components in the feature space.

After constructing the monitoring statistics, the confidence limits of the two statistics need to be determined. However, the process variables are not necessarily mutually independent and obey Gaussian distribution. Hence, the traditional selected method of calculating the confidence limits is not applicable any longer in KECA. Here, KDE method is introduced to determine the confidence limits of the two statistics. The PDFs of $T^{2}$ and SPE statistics based on sample data of the normal condition are first obtained. The area surrounded by the PDF curve and $x$-axis is 1 . The statistical confidence $\beta$ is selected to determine the confidence limits of $T^{2}$ and SPE statistics. The confident limits of the two statistics can be formulated as follows:

$$
\begin{array}{r}
\int_{T_{\lim }^{2}}^{+\infty} \gamma_{T^{2}}=1-\beta, \\
\int_{\mathrm{SPE}_{\lim }}^{+\infty} \gamma_{\mathrm{SPE}}=1-\beta .
\end{array}
$$




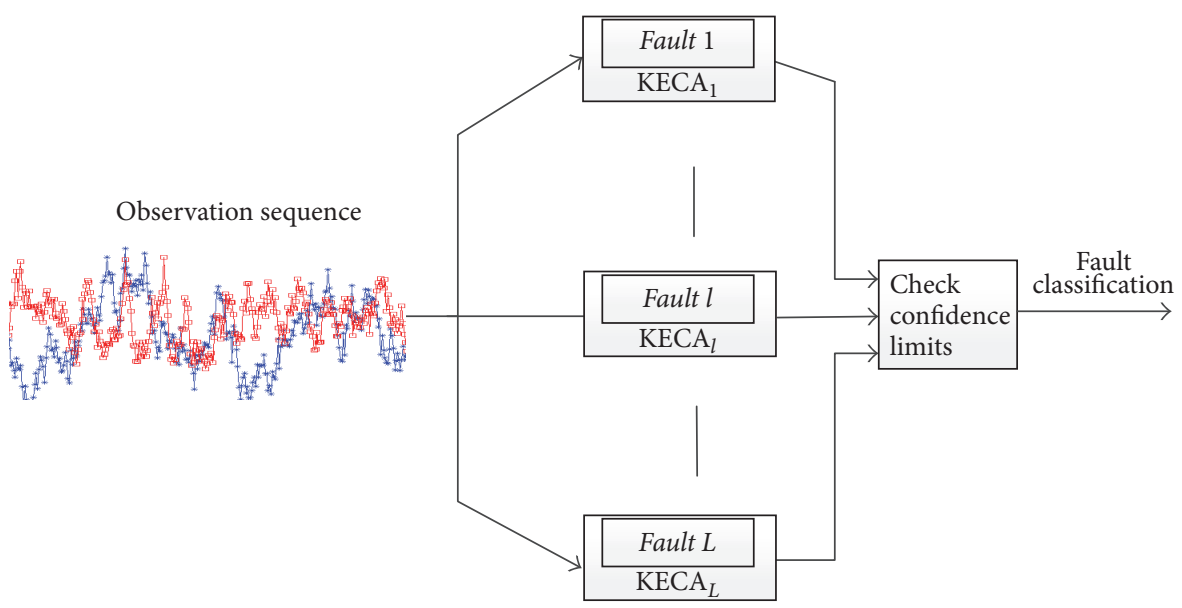

FIGURE 1: Architecture of the KECA-based fault detection algorithm.

The architecture of the KECA-based fault detection algorithm is shown in Figure 1.

4.2. SVM Algorithms for Fault Classification. Support vector machine (SVM) is adopted to identify the faults. For the classification of two-class samples, denote the training set as $D=\left\{\left(\mathbf{x}_{i}, y_{i}\right) \mid i=1,2, \ldots, n\right\}$, where $\mathbf{x}_{i} \in \mathbf{R}^{n}$ stands for $n$ samples and $y_{i} \in\{+1,-1\}$ their class values. Define two standard hyperplanes $H_{1}: \mathbf{w} \cdot \boldsymbol{\Phi}(\mathbf{x})+b=+1$ and $H_{2}: \mathbf{w} \cdot \boldsymbol{\Phi}(\mathbf{x})+b=-1$, where $\mathbf{w}$ is the weight vector and $b$ the bias. The distance between two standard hyperplanes is called classification interval. The optimal hyperplane is a hyperplane $H: \mathbf{w} \cdot \boldsymbol{\Phi}(\mathbf{x})+b=0$ that can correctly separate the two-class samples and the classification interval has the maximum value. As a result, two standard hyperplanes include the samples that are closest to the optimal hyperplane. To maximize the classification interval, $\|\mathbf{w}\|^{2}=\mathbf{w}^{T} \mathbf{w}$ should be minimized because the distance between $H_{1}$ and $H_{2}$ is $2 /\|\mathbf{w}\|$. To ensure that there is not any sample between $H_{1}$ and $\mathrm{H}_{2}$, the classification plane should satisfy the following constraint:

$$
y_{i}\left[\mathbf{w} \cdot \boldsymbol{\Phi}\left(\mathbf{x}_{i}\right)+b\right] \geq 1, \quad i=1,2, \ldots, n .
$$

To construct the optimal hyperplane when the sample is linearly inseparable, a nonnegative relaxation variable $\xi_{i} \geq 0$ is introduced. Then the constraint of the classification plane is changed into

$$
y_{i}\left[\mathbf{w} \cdot \Phi\left(\mathbf{x}_{i}\right)+b\right] \geq 1-\xi_{i}, \quad i=1,2, \ldots, n .
$$

These samples which make the equality in (15) hold are called support vectors. To construct the optimal classification plane requires solving the following optimization problem:

$$
\begin{array}{ll}
\min & \frac{1}{2} \mathbf{w}^{T} \mathbf{w}+C \sum_{i=1}^{n} \xi_{i}, \\
\text { s.t. } & y_{i}\left[\mathbf{w} \cdot \boldsymbol{\Phi}\left(\mathbf{x}_{i}\right)+b\right] \geq 1-\xi_{i}, \quad i=1,2, \ldots, n,
\end{array}
$$

where $C$ is the penalty parameter that compromises the minimum falsely separated samples and the maximum classification interval. By using Lagrange function, the above problem can be transformed into

$$
\begin{aligned}
L(\mathbf{w}, b, \xi, \alpha, \beta)= & \frac{1}{2} \mathbf{w}^{T} \mathbf{w}+C \sum_{i=1}^{n} \xi_{i} \\
& -\sum_{i=1}^{n} \alpha_{i}\left[y_{i}\left(\mathbf{w} \cdot \boldsymbol{\Phi}\left(\mathbf{x}_{i}\right)+b\right)+\xi_{i}-1\right] \\
& -\sum_{i=1}^{n} \beta_{i} \xi_{i},
\end{aligned}
$$

where $\alpha_{i}$ and $\beta_{i}$ are Lagrange multipliers. By calculating partial derivatives with respect to $\mathbf{w}, \xi_{i}, b$ and making them equal to zero, the following equations can be obtained:

$$
\begin{aligned}
& \frac{\partial L}{\partial \mathbf{w}}=0 \Longrightarrow \mathbf{w}=\sum_{i=1}^{n} \alpha_{i} y_{i} \Phi\left(\mathbf{x}_{i}\right), \\
& \frac{\partial L}{\partial b}=0 \Longrightarrow \sum_{i=1}^{n} \alpha_{i} y_{i}=0, \\
& \frac{\partial L}{\partial \xi_{i}}=0 \Longrightarrow C-\alpha_{i}-\beta_{i}=0 .
\end{aligned}
$$

Since $\alpha_{i} \geq 0, \beta_{i} \geq 0$, and $C-\alpha_{i}-\beta_{i}=0,0 \leq \alpha_{i} \leq C$ can be obtained. Substituting (18) into (17) results in

$$
\begin{aligned}
L & (\mathbf{w}, b, \xi, \alpha, \beta) \\
& =\sum_{i=1}^{n} \alpha_{i}-\frac{1}{2} \sum_{i=1}^{n} \sum_{j=1}^{n} \alpha_{i} \alpha_{j} y_{i} y_{j}\left[\Phi\left(\mathbf{x}_{i}\right) \cdot \Phi\left(\mathbf{x}_{j}\right)\right] .
\end{aligned}
$$

Based on Mercer condition, inner product $\left[\boldsymbol{\Phi}\left(\mathbf{x}_{i}\right) \bullet\right.$ $\Phi\left(\mathbf{x}_{j}\right)$ ] in feature space can be replaced by kernel function 


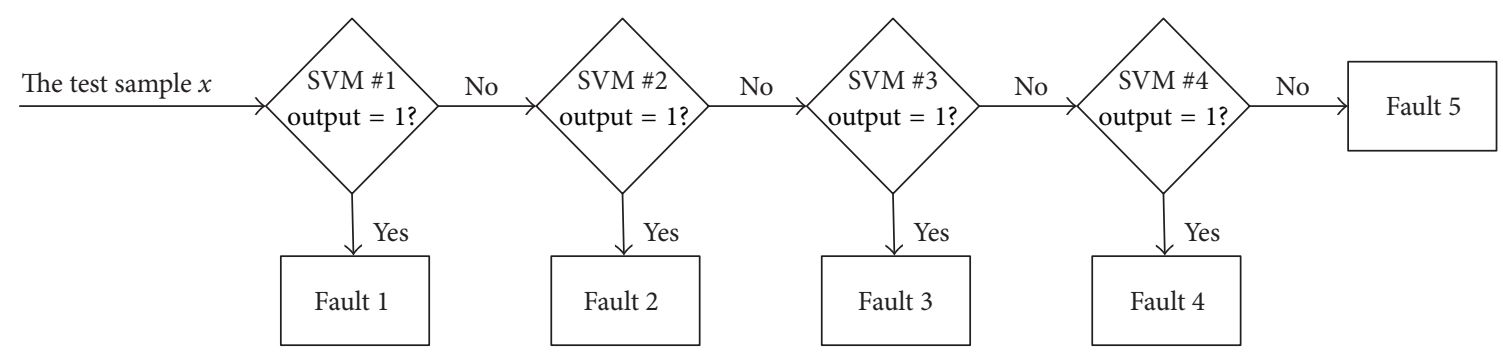

FIGURE 2: Architecture of the SVM-based fault classification algorithm.

$K\left(\mathbf{x}_{i}, \mathbf{x}_{j}\right)$; it can then be transformed into the dual quadratic programming problem as follows:

$$
\begin{array}{ll}
\max _{\alpha} & \sum_{i=1}^{n} \alpha_{i}-\frac{1}{2} \sum_{i=1}^{n} \sum_{j=1}^{n} \alpha_{i} \alpha_{j} y_{i} y_{j} K\left(\mathbf{x}_{i}, \mathbf{x}_{j}\right), \\
\text { s.t. } & \sum_{i=1}^{n} y_{i} \alpha_{i}=0 \quad 0 \leq \alpha_{i} \leq C, i=1,2, \ldots, n .
\end{array}
$$

After working out $\alpha_{i}$, given the test samples $\mathbf{x}$, classification function of SVM classifier can then be written as follows:

$$
\begin{aligned}
f(\mathbf{x}) & =\operatorname{sgn}[\mathbf{w} \cdot \boldsymbol{\Phi}(\mathbf{x})+b] \\
& =\operatorname{sgn}\left[\sum_{i=1}^{n} \alpha_{i} y_{i} K\left(\mathbf{x}_{i}, \mathbf{x}\right)+b\right],
\end{aligned}
$$

where sgn[ ] is the sign function. The class to which $\mathbf{x}$ belongs can be determined by the positive and negative sign of the classification function $f(\mathbf{x})$.

It can be known that SVM classifier is a typical twoclass classifier. However, there are more than two kinds of faults in HTGR. As a result, multiclass SVM classifier should be adopted. Usually, there are two methods to construct multiclass SVM classifier. One is the multiclass classification algorithm put forward by Weston in 1998. This method reconstructs multiclass classification model and chooses the new objective function of optimization. But the objective function of this algorithm is very complicated due to many variables; it greatly increases the difficulty of solving optimization problem and decreases the classification accuracy. Another method is to combine several two-class classifiers.

The architecture of the SVM-based fault classification algorithm is shown in Figure 2. When constructing classifier $m$, the output of Class $m$ samples is trained as 1 and the output of the rest of samples is trained as -1 . The test sample $x$ is first put into classifier 1 . If the output of classification function $f^{1}(x)$ is $1, x$ belongs to Class 1 . Otherwise, $x$ is put into classifier 2. If the output of $f^{2}(x)$ is $1, x$ belongs to Class 2. Otherwise, $x$ is put into classifier 3 . The rest can be done in the same manner. When $x$ is put into classifier $k-1$, if the output of $f^{k-1}(x)$ is $1, x$ belongs to Class $k-1$. Otherwise, $x$ belongs to Class $k$.

This paper is first proposing the algorithms combining KECA and SVM to solve the fault diagnosis problems of HTGR. The numerical experiments of KECA-SVM algorithm are expected to be performed in the future work.

\section{Conclusion}

The wireless sensor network can not only reduce the cost of regular monitoring but also enable the ability to achieve intelligent monitoring, which could enhance the economic competitiveness of HTGR. The intelligent monitoring schemes for three example applications of the condition-based maintenance, the fault diagnosis and recovering, and the management of spent fuel materials are proposed. Three major concerns about applying wireless technology in NPPs are addressed. By comparison, the Wi-Fi technology is promising to be the underlying platform for wireless data communication in HTGR. Statistical data processing algorithms, KECASVM-based algorithms for fault detection and identification, are developed. The numerical experiments of KECA-SVM algorithm are expected to be performed in the future work.

\section{Conflicts of Interest}

The authors declare that there are no conflicts of interest regarding the publication of this paper.

\section{Acknowledgments}

This paper is jointly supported by the National Natural Science Foundation of China (Grant no. 61502270) and the National S\&T Major Project (Grant no. ZX06901).

\section{References}

[1] IAEA, Advanced Surveillance, Diagnostic and Prognostic Techniques in Monitoring Structures, Systems and Components in Nuclear Power Plants, International Atomic Energy Agency, 2013.

[2] J. Li, X. Kang, Z. Long, J. Meng, and X. Huang, "The application of the wireless sensor network in intelligent monitoring of nuclear power plants," in Nuclear Power Plants: Innovative Technologies for Instrumentation and Control Systems, vol. 400, pp. 179-188, Springer, Singapore, 2016.

[3] S. J. Qin, "Survey on data-driven industrial process monitoring and diagnosis," Annual Reviews in Control, vol. 36, no. 2, pp. 220-234, 2012.

[4] H. M. Hashemian, "Wireless sensors for predictive maintenance of rotating equipment in research reactors," Annals of Nuclear Energy, vol. 38, no. 2-3, pp. 665-680, 2011.

[5] Z. Zhang, Y. Dong, F. Li et al., "The shandong shidao Bay 200 MWe high-temperature gas-cooled reactor pebble-bed 
module (HTR-PM) demonstration power plant: an engineering and technological innovation," Engineering, vol. 2, no. 1, pp. 112118, 2016.

[6] "International Electrotechnical Commission: Electromagnetic compatibility (EMC)-Part 4-3: Testing and measurement techniques-Radiated, radio-frequency, electromagnetic field immunity test (IEC 61000-4-3)," 2010.

[7] United States Nuclear Regulatory Commission, Guidelines for Evaluating Electromagnetic and Radio-Frequency Interference in Safety-Related Instrumentation and Control Systems (Regulatory Guide 1.180), 2003.

[8] D. Trask, C. Jung, and M. MacDonald, "Cyber security for remote monitoring and control of small reactors," in Proceedings of the 19th Pacific Basin Nuclear Conference (PBNC '14), Vancouver, Canada, 2014.

[9] S. Jiang, B. Chai, Y. Cao, S. Zhai, and X. Deng, "Study of the technical solution to the dedicated wireless communication system in nuclear power plant," Chinese Journal of Nuclear Science and Engineering, vol. 30, pp. 275-279, 2010 (Chinese).

[10] J. M. Lee, C. K. Yoo, S. W. Choi, P. A. Vanrolleghem, and I. B. Lee, "Nonlinear process monitoring using kernel principal component analysis," Chemical Engineering Science, vol. 59, no. 1, pp. 223-234, 2004.

[11] I. Elaissi, I. Jaffel, O. Taouali, and H. Messaoud, "Online prediction model based on the SVD-KPCA method," ISA Transactions, vol. 52, no. 1, pp. 96-104, 2013.

[12] V. H. Nguyen and J. C. Golinval, "Fault detection based on Kernel Principal Component Analysis," Engineering Structures, vol. 32, no. 11, pp. 3683-3691, 2010.

[13] Y. W. Zhang and C. Ma, "Fault diagnosis of nonlinear processes using multiscale KPCA and multiscale KPLS," Chemical Engineering Science, vol. 66, no. 1, pp. 64-72, 2011.

[14] L. V. Gan, H. Z. Cheng, H. B. Zhai, and L. X. Dong, "Fault diagnosis of power transformer based on multi-layer SVM classifier," Electric Power Systems Research, vol. 7, no. 1, pp. 1-7, 2005.

[15] K. C. Gryllias and I. A. Antoniadis, "A Support Vector Machine approach based on physical model training for rolling element bearing fault detection in industrial environments," Engineering Applications of Artificial Intelligence, vol. 25, no. 2, pp. 326-344, 2012.

[16] J. Cui and Y. Wang, "A novel approach of analog circuit fault diagnosis using support vector machines classifier," Measurement, vol. 44, no. 1, pp. 281-289, 2011.

[17] Z. Chenglin, S. Xuebin, S. Songlin, and J. Ting, "Fault diagnosis of sensor by chaos particle swarm optimization algorithm and support vector machine," Expert Systems with Applications, vol. 38, no. 8, pp. 9908-9912, 2011. 


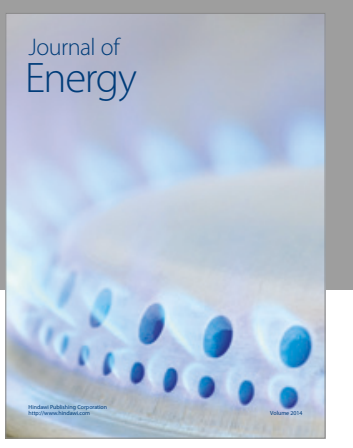

Journal of

Industrial Engineering
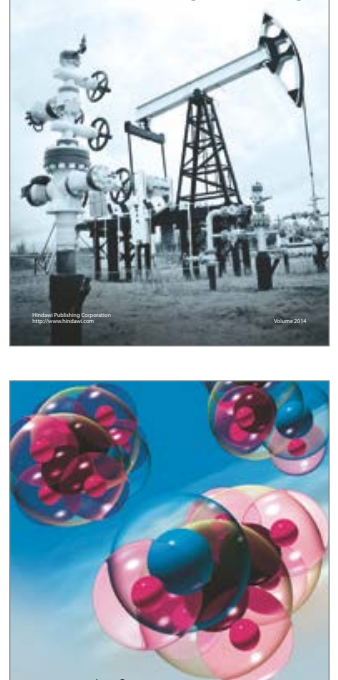

Fuels
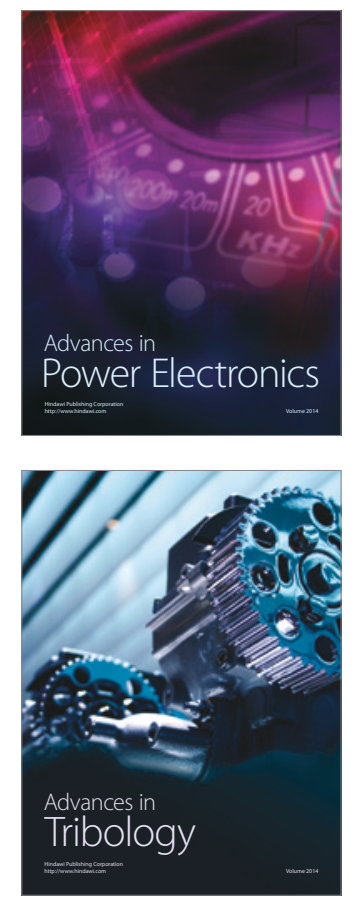
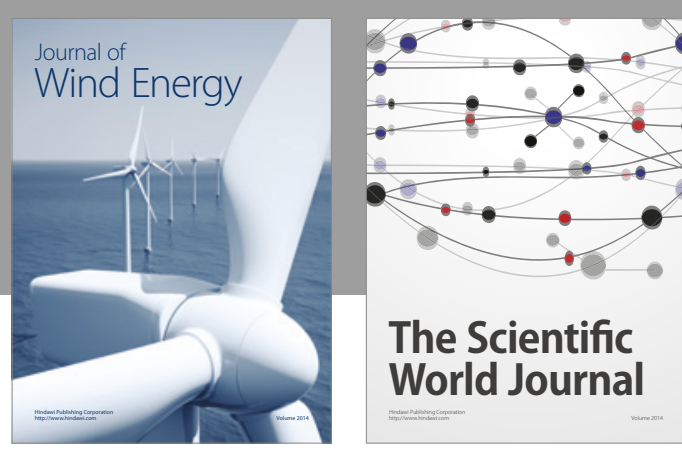

The Scientific World Journal
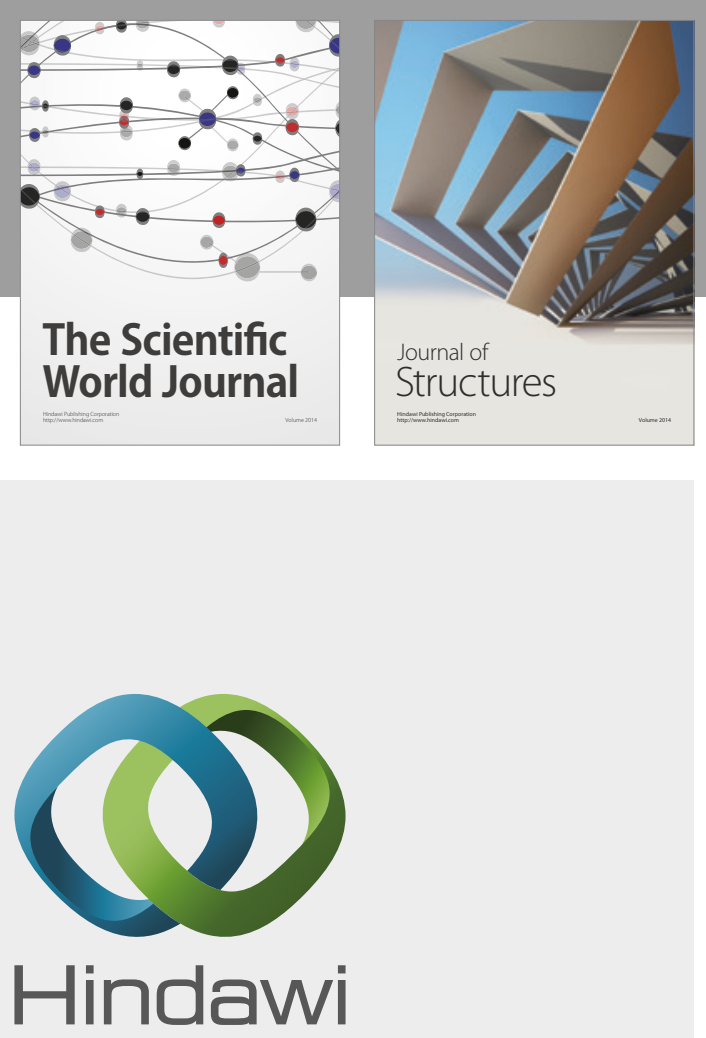

Submit your manuscripts at

https://www.hindawi.com
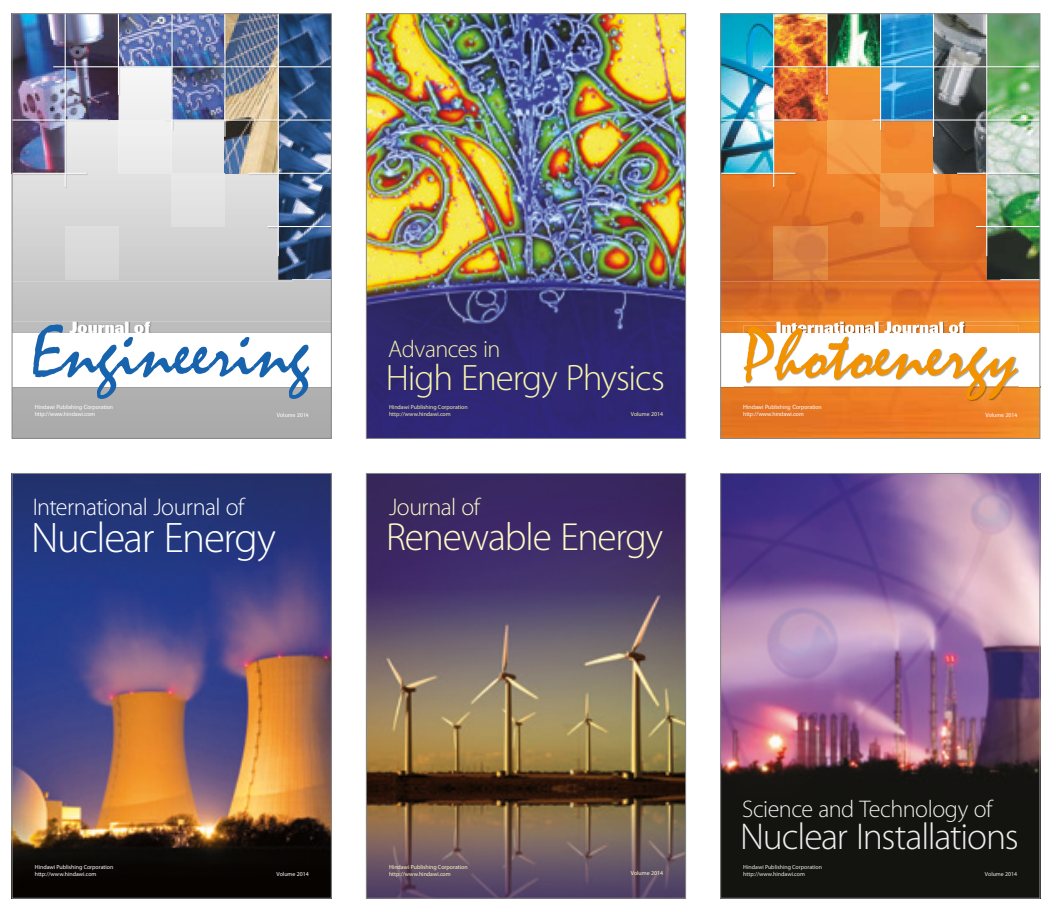

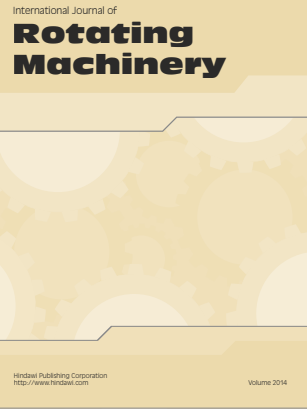

Journal of

Petroleum Engineering

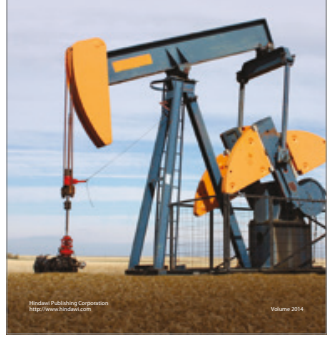

Journal of
Solar Energy
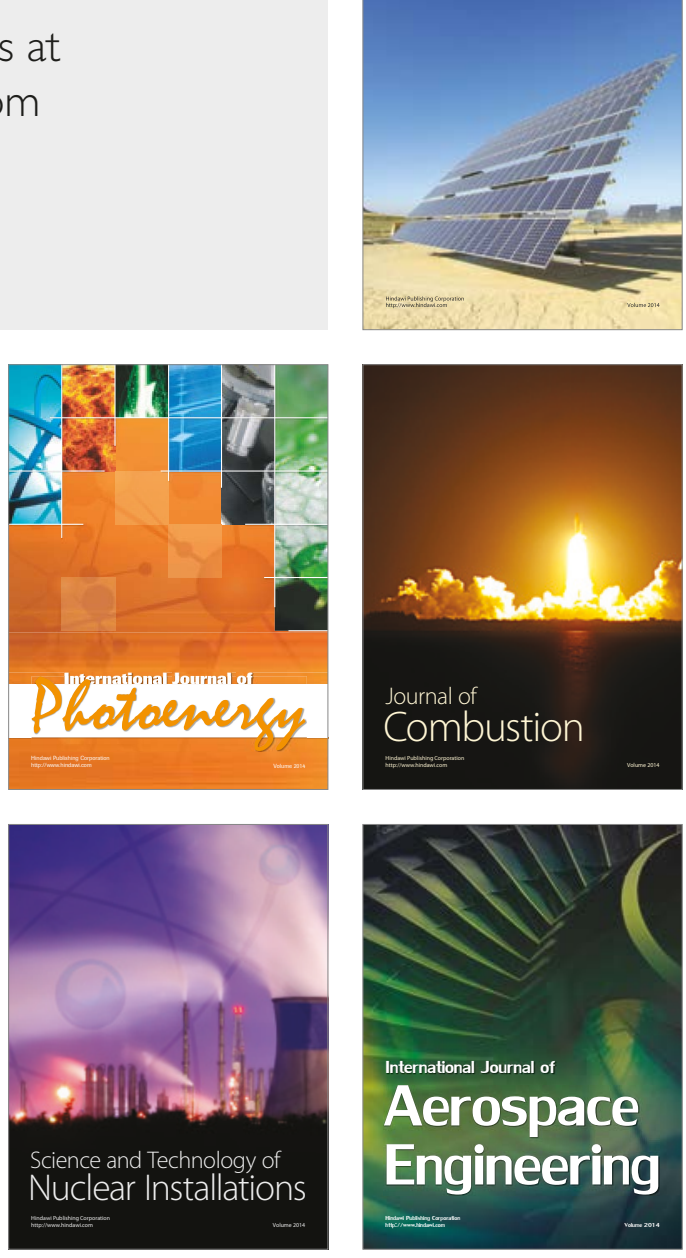